\title{
Interference with the Mate-Finding Communication System of the Obliquebanded Leafroller (Lepidoptera: Tortricidae) Using Synthetic Sex Pheromones
}

\author{
D. S. LAWSON, W. H. REISSig, A. M. AGNELLO, J. P. NYROP, AND W. L. ROELOFS \\ Department of Entomology, Comell University, New York State Agricultural Experiment Station, \\ Geneva, NY 14456
}

\begin{abstract}
Environ. Entomol. 25(5): 895-905 (1996)
ABSTRACT Effect of atmospheric permeation with synthetic sex pheromone on the behavior and control of obliquebanded leafroller, Choristoneura rosaceana (Harris), was studied in small and large plots in commercial apple orchards. Synthetic sex pheromone significantly reduced the ability of male moths to locate pheromone-baited traps and tethered females; however, no differences were found among 3 pheromone rates. Location of pheromone dispensers within the tree canopy did not alter the number of males locating pheromone-baited traps and mating tethered females. Obliquebanded leafroller activity was greatest in the upper positions of the tree canopy and no edge effect was observed around perimeters of large disnupted blocks. The presence of gravid feral females, mated tethered females, high larval densities, and fnit damage within large pheromone disrupted blocks indicate obliqubanded leafrollers mate in orchards treated with synthetic sex pheromone. However, fruit damage caused by obliquebanded leafroller larvae was similar in pheromone, pheromone plus insecticide, and insecticide treatments.
\end{abstract}

KEY WORDS Choristoneura rosaceana, mating disruption, apple, sex pheromone, traps, behavior

THE OBLIQUebanded LEAFroller, Choristoneura rosaceana (Harris), is a pest of apples throughout North America. In western New York State, it damages as much as $20 \%$ of the fnuit in some orchards. During the mid-1960s, the obliquebanded leafroller was considered only a foliage feeder of apple (Chapman et al. 1968, Chapman and Lienk 1971). By the mid-1970s, obliquebanded leafrollers were causing "some significant fruit damage" to apple plantings despite multiple insecticide applications (Reissig 1978). During the 1980s, obliquebanded leafrollers became a more serious pest and a phenological model was developed to allow better timing of insecticide applications (Onstad et al. 1985). At the same time, Reissig et al. (1986) documented that this pest was becoming resistant to insecticides. The resulting damage caused by obliquebanded leafrollers makes it important to evaluate alternative integrated pest management control strategies.

One promising alternative to insecticides for control of tortricid pests is the use of synthetic sex pheromones to disrupt their mate-finding communication systems (Ridgway et al. 1990). Sex pheromones of the major tortricid pests of tree fruits have been identified and used in mating disruption experiments. Mating disruption of the codling moth, Cydia pomonella (L.), in apple and pear orchards produced encouraging results (Mof- fitt and Westigard 1984, Barnes et al. 1992, Howell et al. 1992, Pfeiffer et al. 1993b). Mating disruption of the oriental fruit moth, Grapholita molesta (Busck), redbanded leafroller, Argyrotaenia velutinana (Walker), tufted apple bud moth, Platynota ideausalis (Walker), and the variegated leafroller $P$. flavedana (Clemens) also has been studied. Some of these studies only examined the ability of pheromone permeation to prevent males from locating pheromone-baited traps, whereas others were large scale trials that measured fruit damage (Cardé et al. 1975, Rothschild 1975, Roelofs and Novak 1981, Weakely et al. 1987, Pfeiffer et al. 1993a). Results of these studies have indicated the potential of mating disruption as a control strategy, as well as our inadequacies in understanding how this communication system works and the factors that affect successful use of mating disruption.

In eastern North America the obliquebanded leafroller sex pheromone consists of (Z)-11-tetradecen-1-yl acetate (Z11-14:OAc), (E)-11-tetradecen-1-yl acetate (E11-14:(OAc), and (Z)-11-tetradecen-1-ol (Z11-14:OH) (Roelofs and Tette 1970, Hill and Roelofs 1979) and pheromone dispensers containing this blend are available (Shin-Etsu, Tokyo, Japan, and Consep, Bend, OR). Use of sex pheromone permeation to interfere with obliquebanded leafroller mate finding was first attempted by Reissig et al. (1978). They were able to reduce 
the ability of the male obliquebanded leafroller to locate pheromone-baited traps. Recently, Deland et al. (1994) also demonstrated that male obliquebanded leafrollers were less likely to locate pheromone-baited traps in the presence of synthetic sex pheromone. However, no research has been conducted on a large enough scale to determine the effect of atmospheric permeation with synthetic sex pheromone as a control strategy for obliquebanded leafrollers and the commercial feasibility of this technique. Additional knowledge of obliquebanded leafroller behavior in large scale pheromone disruption trials is vital for the most efficient use of mating disruption techniques (Roelofs and Novak 1981).

This artical reports on small and large plot pheromone disruption studies conducted during a 2-yr period. The objectives of these studies were to determine the effect of altering pheromone release rate and pheromone dispenser deployment on male and female obliquebanded leafroller behavior, whether the edges of pheromone-disrupted plots have increased obliquebanded leafroller activity and fruit damage, the effect of mating disruption on obliquebanded leafroller behavior in large apple plantings and its effectiveness as a control strategy, and the effect of combining synthetic sex pheromone and insecticides for control of obliquebanded leafrollers.

\section{Materials and Methods}

To thoroughly evaluate pheromone permeation techniques, multiple monitoring methods must be used. These monitoring methods need to measure the ability of males to locate and mate with females, the number of gravid females within pheromone-permeated blocks, and crop damage caused by the targeted insect (Rothschild 1975, Marks 1976, Charlton and Cardé 1981, Rothschild 1981, Kehat et al. 1985). Therefore, we chose to integrate data from pheromone and passive traps, tethered females, larval density estimates, and fruit damage as a method for evaluating the effect of sex pheromone permeation on obliquebanded leafroller behavior and control.

Mating disruption studies were conducted during 1993 and 1994 in 3 commercial apple orchards in western New York State. Orchards were chosen that were representative of commercial apple orchards in this region and had a history of high obliquebanded leafroller densities. Small-plot studies were used to measure the effect of pheromone permeation rates and the effect of pheromone dispenser placement on obliquebanded leafroller behavior. Results from these studies were used to conduct large-plot disruption studies. Large plots were necessary to evaluate the effectiveness of pheromone permeation, because they reduce the impact of immigrating moths, allow thorough mixing of pheromone within the orchard, ensure that moths within the disrupted block were sufficiently exposed to the pheromone, facilitate larval and fruit sampling, and measure pheromone disruption techniques under commercial conditions.

1993: Small-Plot Trials. Orchard 1, located in Niagara County, contained 'Cortland' and 'Idared' on semidwarf rootstocks, 4.7-5.4 m high planted at a density of 320 trees per hectare. Orchard 2, located in Orleans County, contained Idared and Cortland on semidwarf rootstocks at a density of 341 trees per hectare and a tree height of $4.4-5.5$ $\mathrm{m}$. Orchard 3, also located in Orleans County, consisted of Red Delicious and Cortland at a density of 287 trees per hectare on semidwarf rootstocks and had a tree height of $4.9-5.5 \mathrm{~m}$.

Within each orchard, 3 (0.2-ha) and 4 (0.04-ha) plots were established. All plots were separated by at least $40 \mathrm{~m}$. Synthetic obliquebanded leafroller sex pheromone incorporated into polyethylene rope type dispensers (Shin-Etsu.) supplied by $\mathrm{Pa}$ cific Biocontrol, Davis, CA, were affixed to all trees within each plot by twisting the dispensers around a branch. Analyses of the dispensers using gas chromatography indicated that dispensers contained 83\% Z11-14:OAc, 8\% E11-14:OAc, and 9\% Z11-14:OH. Manufacturer specifications indicated that each dispenser contained $80.2 \mathrm{mg}$ (AI) and had a release rate of $\approx 0.025 \mathrm{mg} / \mathrm{h}$.

Pheromone Concentration Study. The effect of atmospheric pheromone concentration on the ability of male obliquebanded leafrollers to locate pheromone-baited traps was tested in the 0.04-ha plots. In all treatments, equal numbers of pheromone dispensers were placed near the top and bottom of the tree canopy. The 4 treatments were as follows: (1) 990 dispensers per hectare releasing pheromone at a rate of $24.8 \mathrm{mg} / \mathrm{h} / \mathrm{ha}$; (2) 1,980 dispensers per hectare releasing pheromone at a rate of $49.5 \mathrm{mg} / \mathrm{h} / \mathrm{ha}_{\text {; }}$ (3) 2,970 dispensers per hectare releasing pheromone at a rate of $74.3 \mathrm{mg} / \mathrm{h} / \mathrm{ha}$; and (4) no pheromone. A 2-tier pheromone trap was placed in the center of each plot. This trap was composed of 2 winged Pherocon 1C traps (Trécé, Salinas, CA) attached to a conduit pipe. The low trap was attached $1 \mathrm{~m}$ above the ground and the high trap was attached $3.0 \mathrm{~m}$ above the ground to the same pipe. Each trap was baited with a standard rubber septum lure impregnated with $0.485 \mathrm{mg}$ Z11-14:OAc, 0.015 mg E11-14:OAc, and $0.025 \mathrm{mg} \mathrm{Z11-14:OH} \mathrm{(Trécé).}$

Pheromone Dispenser Position Study. The effect of pheromone dispenser placement within the tree canopy on obliquebanded leafroller mating and behavior was examined in the 0.2-ha plots. The 3 treatments evaluated were as follows: (1) pheromone dispensers placed near the top of the canopy releasing pheromone at a rate of 24.8 $\mathrm{mg} / \mathrm{h} / \mathrm{ha}$ (990 dispensers per hectare); (2) equal numbers of pheromone dispensers placed near the top and bottom of the tree canopy releasing pheromone at a rate of $24.8 \mathrm{mg} / \mathrm{h} / \mathrm{ha}$ (990 dispensers per hectare), and; (3) no pheromone. A 2-tier pheromone trap was placed in the center of each 
plot. This trap was of the same design as described in the pheromone concentration study. Two-tier passive traps were placed near the 4 corners of each large plot. These traps consisted of clear 3.5 $\mathrm{mm}$ thick Plexiglas panels $(0.61$ by $0.61 \mathrm{~m})$ attached to the lowest scaffold branch, $\approx 0.5-1 \mathrm{~m}$ above the ground and another trap attached to the top scaffold branch, $\approx 3-4 \mathrm{~m}$ above the ground in the same tree. Both sides of the traps were coated with a thin layer of STP oil treatment (First Brands, Danbury, CT) to intercept obliquebanded leafrollers. Passive traps offered a novel approach for evaluating male and female presence within the tree canopy without altering their behavior. These traps were not attractive, therefore animals captured on them were located in the same tree strata as the traps (Weissling and Knight 1994). Additionally, these traps allowed estimates of the mating status of feral females. Bait traps have been used to measure these parameters, but because these traps provide a food source, they are likely to be attractive, particularly to gravid females (Rothschild 1975, Weissling and Knight 1994).

Tethered virgin female obliquebanded leafrollers were used to assess mating among the treatments. Over-wintering and summer brood obliquebanded leafroller larvae were collected from field populations and transferred onto pinto bean dièt, modified from Shorey and Hale (1965), and held in an insectary at ambient temperature and light conditions until pupation. Two-day-old virgin females were chosen for tethering because this age class has been shown to be most attractive to males and posses high titers of sex pheromone (Delisle 1992, 1995; Delisle and Royer 1994). Scales on the pronotum of the female were gently removed with a scalpel and a fine monofilament line was attached with fingernail polish. Hathaway (1979) placed tethered codling moth females in pheromone traps to monitor mating activity. We modified his technique by attaching tethered females to plywood boards $(0.3$ by $0.3 \mathrm{~m}$ ) attached to scaffold branches $\approx 2 / 3$ the distance of the branch from the tree trunk. One board was placed high in the canopy, $\approx 3-4 \mathrm{~m}$ above the ground, and the other was placed low in the canopy of the same tree, $\approx 1 \mathrm{~m}$ above the ground. Three sets of 2-tier mating boards were placed in 3 trees in each treatment per orchard.

During both moth flights, equal numbers of tethered females were placed in each canopy location and in each treatment in 1 orchard per sample night. During the 1st moth flight period (22 June-2 July), 94 females were deployed into 2 orchards. During the 2nd flight period (4-17 August), 96 females were deployed into 1 orchard. Orchards were chosen to be monitored with tethered females when pheromone-baited traps indicated high levels of moth activity. Tethered females, still alive after $24 \mathrm{~h}$ in the orchard, were removed and placed in alcohol in vials for later evaluation of mating status. This was determined by removing the bursa copulatrix and examining it for the presence of a spermatophore.

All pheromone dispensers in small-plot studies were deployed on 2 and 3 June 1993. All traps were checked 2-3 times weekly from 14 June to 8 July and from 2-25 August and the number and sex of obliquebanded leafrollers captured was recorded. Female obliquebanded leafrollers captured on passive traps were removed and placed in alcohol in vials for later determination of mating status. Adhesive in the pheromone traps and pheromone septa were replaced on 30 July. New oil was applied with small paint rollers to passive traps every time they were checked. Within all 3 orchards, growers continued to use their standard pesticide regimes.

1994: Large-Block Pheromone Permeation Studies. The 3 apple orchards used in this study were located in Orleans County New York. Orchard 1 contained Red Delicious, Cortland, and Golden Delicious at a density of 287 trees per hectare. All trees were planted on semidwarf root stocks and had a height of 4.9-5.5 m. Orchard 2 contained 'Monroe', 'Webster', 'Spijon', 'Mutsu' and '20-Ounce' at a density of 250 trees per hectare. Trees were planted on semidwarf rootstocks and had a height of 5.5-6.0 m. Orchard 3 contained 'Rome', Mutsu, Webster, Spijon, and Monroe planted at a density of 490 trees per hectare. These semidwarf trees were $4.2-5.0 \mathrm{~m}$ high.

Within each of the 3 orchards, two 4.1-ha blocks containing the same cultivars were established. Obliquebanded leafroller sex pheromone dispensers (Consep) were attached near the top of all trees in 1 block per orchard before the beginning of moth flight. Analyses of the dispensers using gas chromatography indicated that they contained 92.4\% Z11-14:OAc, 3.6\% E11-14:OAc, and 4\% Z11-14:OH. Specification provided by the manufacture indicated each dispenser contained $200 \mathrm{mg}$ (AI) and had a release rate of $\approx 0.097 \mathrm{mg} / \mathrm{h}$. Dispensers were deployed at a rate of $495 / \mathrm{ha}$, resulting in a pheromone release rate of $48.0 \mathrm{mg} / \mathrm{h} / \mathrm{ha}$.

Pheromone disrupted blocks were subdivided into 3 plots. The center plot did not receive any insecticide applications after petal fall and will be referred to as the pheromone treatment. The 2 remaining plots, on either side of the pheromone treatment, received the same insecticides at petal fall as the pheromone treatment and after petal fall, received the same insecticides as the grower standard and will be referred to as the pheromone plus insecticide treatment. This design allowed for a comparison of pheromone, pheromone plus insecticide, and insecticide alone on obliquebanded leafroller densities and damage.

Pheromone-permeated blocks received insecticide applications of Bacillus thuringiensis (Dipel $2 \times$, Abbott, North Chicago, IL), $0.14 \mathrm{~kg}$ (AI)/ha and chlorpyrifos (Lorsban 50\% WP, [wettable powder], DowElanco, Indianapolis, IN), $1.6-3.8 \mathrm{~kg}$ (AI)/ha during petal fall (27 May-3 June) to reduce 
over-wintering obliquebanded leafroller larvae. The other 4.1-ha block received season-long insecticides for control of obliquebanded leafrollers. Orchard 1 was treated with chlorpyrifos (Lorsban $50 \%$ WP, DowElanco), $3.1 \mathrm{~kg}(\mathrm{AI}) / \mathrm{ha}$ on 2 June and 6, 20 and 28 July. Orchard 2 was treated with chlorpyrifos (Lorsban 50\% WP, DowElanco), 3.8 $\mathrm{kg}(\mathrm{AI}) / \mathrm{ha}$ on 3 June and 6 and 15 July. Orchard 3 was treated with chlorpyrifos (Lorsban $50 \%$ WP, DowElanco), $3.8 \mathrm{~kg}$ (AI)/ha on 27 May and 2, 16 and 29 July and methyl parathion (Penncap-M 2 EC [emulsifiable concentrate], ELF Atochem North America, Philadelphia, PA), $1.3 \mathrm{~kg}$ (AI)/ha on 25 August.

Treatment Effect. In each insecticide and pheromone treatment per orchard, 3 groups of monitoring devices were established to examine the treatment effects on obliquebanded leafroller behavior and control. Each group of monitoring devices contained one 2-tier pheromone trap, one 2-tier passive trap and one 2-tier tethering board. Traps were maintained during the 2 obliquebanded leafroller summer flights as described in the 1993 studies. During peak flight of the 1st summer generation (21 June-1 July) 96 tethered females were deployed into the pheromone and insecticide blocks as previously described.

Obliquebanded leafroller larval densities were estimated on 27 July by counting larvae on the terminals of 10 branches from the tops and 10 from the bottoms of 30 trees in the interior of the pheromone, pheromone plus insecticide and insecticide treatments. Fruit damage at harvest, caused by obliquebanded leafroller larvae was assessed in these treatments by sampling 100 randomly selected apples from the tops and 100 from the bottoms of each of 4 trees in the interior of each treatment. These fruits were rated into four categories (cull, \#1, X-fancy, clean) (New York State Department Agriculture and Marketing 1973).

Edge Effect. To determine if pheromone disruption is less effective near the edges of disrupted orchards, possibly caused by uneven pheromone coverage or immigration of obliquebanded leafrollers from outside the orchard, 1 group of monitoring devices was established in the row of trees along each edge of the disrupted block. During peak flight of the 1st summer generation a total of 46 tethered females were deployed along the edge of pheromone disrupted blocks on the same nights that tethered females were deployed into the blocks interior. Comparisons of trap catch and tethering data from the edge and those in the interior provided information about edge effects. Additional inferences were made by comparing larval densities and fruit damage recorded along the edge and from the interior of pheromone plus insecticide plots. These plots were sampled because they contained the edge rows of the pheromone permeated block and by the time these samples were taken, insecticides had been sprayed onto these plots. Obliquebanded leafroller larval densities were estimated on 27 July from 10 trees, and fruit damage at harvest caused by obliquebanded leafroller larvae was assessed by sampling four trees, along each edge of the pheromone plus insecticide treatment as previously described.

Statistical Analyses. Much of the data recorded in this study was measured from two locations (high and low) within the same tree. Therefore, a repeated measure design was used for the analyses, with the repeated variable being location within tree.

Pheromone Concentration, 1993. Pheromone trap catch from the 1993 pheromone concentration study was transformed using a $\log x+1$ transformation. Transformed data were analyzed using a repeated measures analyses of variance (ANOVA) (Abacus Concepts 1989) with factors of pheromone concentration, trap location and their interaction. Linear contrasts were constructed among all treatments and also among an abridged data set which did not include the control treatment.

Pheromone Dispenser Position, 1993. Pheromone and passive trap data were analyzed using a repeated measures ANOVA with factors of pheromone dispenser location, trap location and the interaction between these effects. Means were compared using Fisher protected least significant difference (LSD) test. Male, female, and mated female moth data from passive traps were analyzed separately. Inspection of the male passive trap data revealed a trend which warranted additional analyses. To examine the difference in location of trap catch within 1 treatment, a separate contrast for location was constructed. This is an unconventional method of analyses; however, we feel that an important biological phenomena is occurring which is difficult to measure and warrants treatment of the data in this manner.

Tethering data were analyzed using logistic regression with treatment effects coded so that mating in pheromone treated plots was contrasted with mating in control plots. Differences in the proportion of mated females among treatments were deemed significant if the $95 \% \mathrm{CI}$ of the odds ratios did not include 1 . Odds ratios are a measure of how likely an outcome is in one treatment compared with another; i.e., the odds of mating in pheromone-disrupted plots compared with insecticide treated plots.

1994: Large-Block Pheromone Permeation Studies. Only trap catch and tethering data from the 1st moth flight (2 June-2 July) in 1994 were used to avoid any confounding effect insecticides applied for summer larvae may have had on subsequent moth flights. Therefore, trap catch data from only the pheromone, pheromone edge, and insecticide plots were used.

Treatment Effects. To examine differences in moth trap catch between disrupted and nondisrupted blocks, trap data was analyzed using a repeated measures ANOVA. Pheromone trap data was transformed using a $\log x+1$ transformation. 


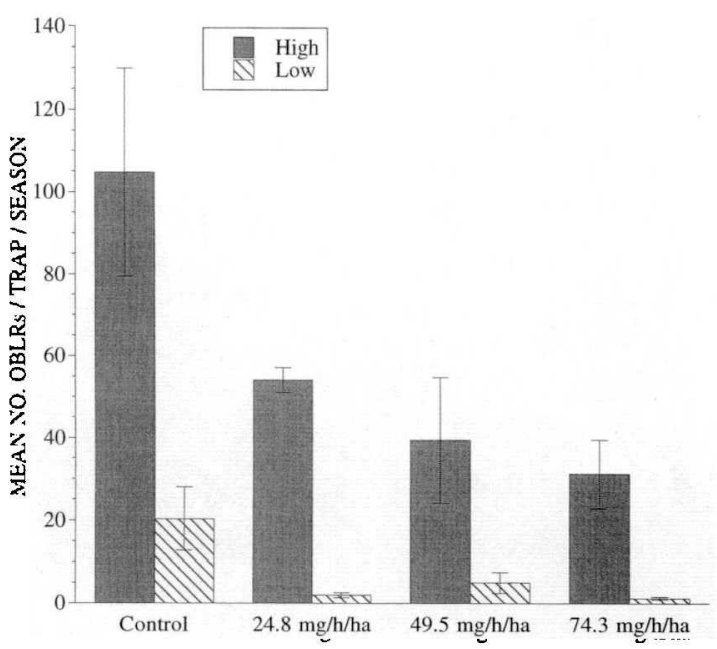

Fig. 1. Effect of atmospheric pheromone concentration on male obliquebanded leafrollers captured in pheromone-baited traps placed high and low in apple tree canopies in 0.04-ha plots, 1993.

Larval densities and fruit damage were examined among the insecticide, pheromone, and pheromone plus insecticide treatments using a repeated measures ANOVA and means were compared using Fisher protected LSD test. Tethering data were analyzed using logistic regression with treatment effects coded so that mating in pheromone treated plots was contrasted with mating in control plots. Differences in the proportion of mated females among treatments were deemed significant if the $95 \% \mathrm{CI}$ if the odds ratios did not include 1.

Edge Effect. The existence of an edge effect along the border of the pheromone disrupted blocks was evaluated by analyzing trap catch data collected along the edge and interior of the pheromone disrupted blocks with a repeated measures ANOVA. Only data collected during the 1st flight period and before any summer insecticide application were used. Fruit damage and percentage of terminals infested with larvae were compared between edge and interior of the pheromone plus insecticide treatment using a repeated measure ANOVA. Tethering data were analyzed as described above, however treatment effects were coded so that mating in the interior of pheromone treated blocks was contrasted with mating along the edge of the same blocks.

\section{Results}

Pheromone Concentration Study, 1993. Synthetic sex pheromone significantly reduced the number of male moths captured in pheromone baited traps $(F=10.59$; $\mathrm{df}=3,16 ; P \leq 0.01)$ and trap catch was diminished in the lower tree canopy compared to the upper canopy $(F=80.92$; $\mathrm{df}=$ $1,16 ; P \leq 0.01)$. There was no interaction between pheromone permeation and trap location. A linear

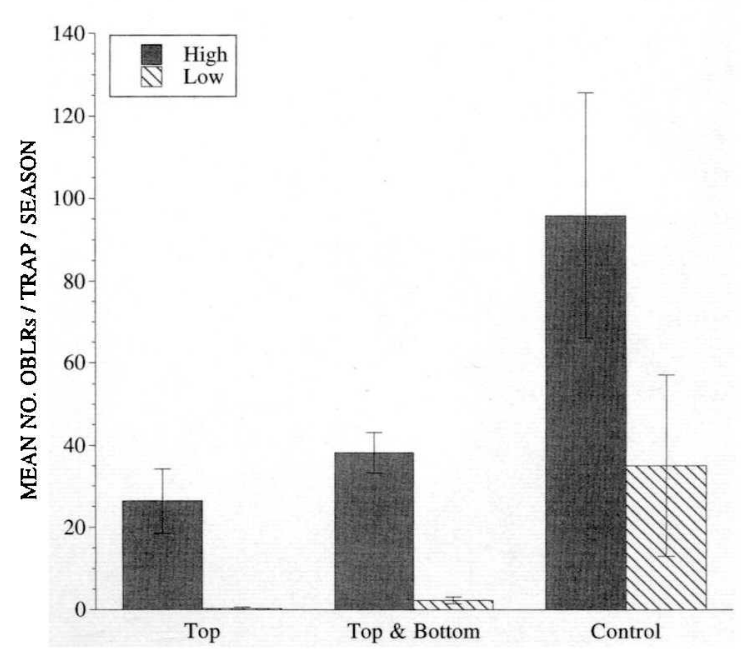

Fig. 2. Effect of pheromone dispenser position (top, top and bottom) within the tree canopy on male obliquebanded leafrollers captured in pheromone-baited traps placed high and low in apple tree canopies in 0.2 -ha plots, 1993.

contrast among the three pheromone concentrations and control was significant $(F=12.47$; $\mathrm{df}=$ 1,$16 ; P \leq 0.01$ ). However, inspection of means (Fig. 1) suggested that the control differed from the 3 pheromone treatments, but there was no difference among the concentrations. A subsequent linear contrast for just the three pheromone concentrations confirmed this as the contrast was not significant.

Pheromone Dispenser Position Study, 1993. Although pheromone dispensers in the top or top and bottom of the tree canopy significantly reduced the number of males captured in pheromone-baited traps compared with the untreated control $(F=25.77 ; \mathrm{df}=2 ; 12 ; P \leq 0.00)$, there were no differences in trap catch reduction between the 2 spatial deployment patterns (Fig. 2). As in the previous study, there were significantly more males captured in traps placed high in the tree canopy compared with those placed low $(F=$ 37.47; $\mathrm{df}=1,12 ; \boldsymbol{P} \leq 0.01$ ). A significant treatment by trap location interaction also occurred and is attributable to the increased difference between trap catches high and low in the tree canopy in pheromone-disrupted treatments compared with the control $(F=5.00 ; \mathrm{df}=2,12 ; P=0.03)$.

The number of male and female obliquebanded leafrollers captured on passive traps did not differ among treatments. However, significantly more females were captured on passive traps located high in the tree canopy in all treatments $(F=8.54$; df $=1,12 ; P=0.01)$. Inspection of the male passive trap data revealed an increased difference between trap catches high and low in the tree canopy in the top and bottom pheromone dispenser treatment compared to the other treatments (Fig. 3). An orthogonal contrast between data from the high and 


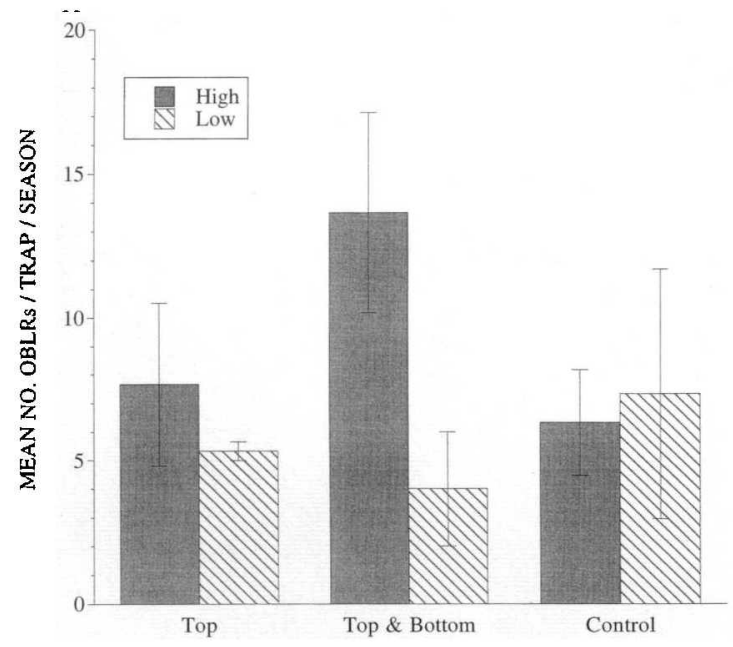

Fig. 3. Effect of pheromone dispenser position (top, top and bottom) within the tree canopy on male obliquebanded leafrollers captured on passive traps placed high and low in apple tree canopies in 0.2-ha plots, 1993.

low traps in the top and bottom dispenser treatment indicate that significantly $(F=5.80 ; \mathrm{df}=1$, 4; $P=0.07$ ) more males were captured in the trap located high compared with low in the tree canopy.

Of the 190 tethered females placed in the field, $77.1 \%$ were recovered alive. Significantly fewer females mated in the pheromone-permeated plots than in the untreated control plots $\left\langle x^{2}=10.23\right.$, df $=2, P \leq 0.01$ ). No females mated in the top dispenser treatment, whereas in the top and bottom dispenser treatment $4.0 \%$ of the females located high in the tree canopy mated. In the control 5.6 and $20.8 \%$ of the tethered females mated in the high and low position respectively.

Treatment Effect, 1994. Significantly fewer males were captured in pheromone-baited traps in pheromone compared to insecticide treated plots $(F=$ 35.97; $\mathrm{df}=1,8 ; P \leq 0.01$ ) and more were captured in traps located high in the canopy compared to low ( $F=26.50$; $\mathrm{df}=1,8 ; P \leq 0.01$ ) (Fig. 4). A significant treatment by trap location interaction was observed and results from a greater difference between trap catches high and low in the tree canopy in the pheromone-disrupted treatment compared to the insecticide treatment $(F=5.79 ; \mathrm{df}=$ 1,$8 ; P=0.04$ ).

Significantly fewer male obliquebanded leafrollers were captured on passive traps in the pheromone-permeated treatment compared with the insecticide treatment $(F=6.14 ; \mathrm{df}=1,8 ; P=0.04)$. However, no differences were observed for the number of males captured on passive traps at different tree canopy locations (Fig. 5). No differences in the number of female or gravid female obliquebanded leafrollers captured on passive traps were observed among the treatments or within the different tree canopy locations.

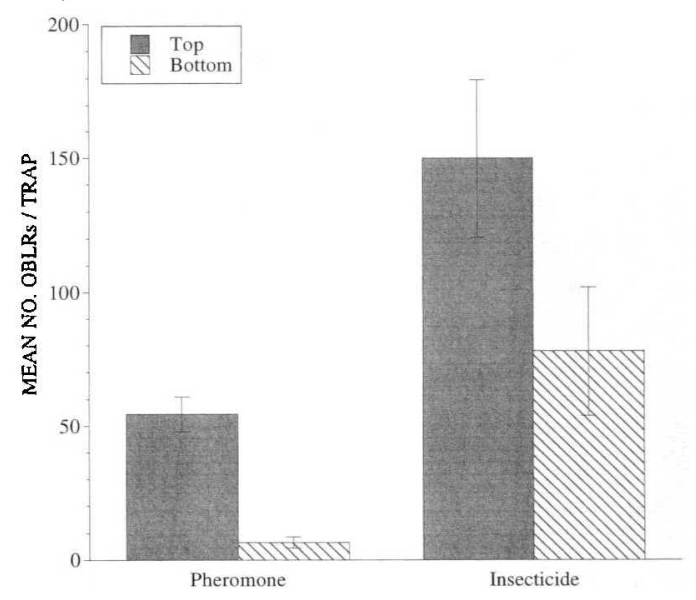

Fig. 4. Male obliquebanded leafrollers captured in pheromone-baited traps placed high and low in apple tree canopies in 4.1-ha pheromone-disrupted and insecticide-treated blocks during the 1st summer flight period, 1994.

Of the 96 tethered females deployed into the pheromone and insecticide treatments during the 1st moth flight, 53.1\%were recovered alive. Mating was significantly greater in the insecticide treatment compared to the pheromone disrupted treatment $\left(\chi^{2}=14.29, \mathrm{df}=2, P \leq 0.01\right.$; odds ratio $=$ $9 \pm 2)$. In the pheromone treatment, 13.3 and $0.0 \%$ of the tethered females in the high and low canopy position respectively, and 36.4 and $46.2 \%$ in the high and low positions respectively in the insecticide treatment, mated. Position of tethered females within the tree canopy (high or low) was not significant.

Percentage of terminal branches infested with obliquebanded leafroller larvae was not signifi-

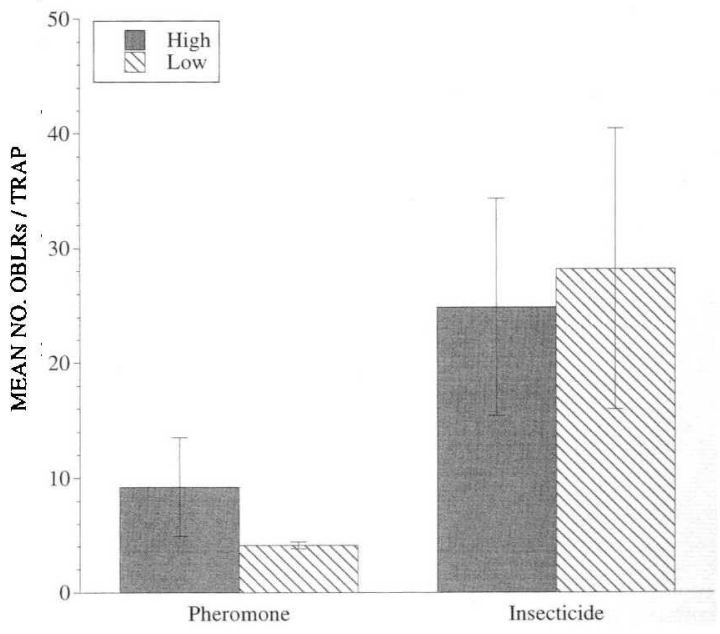

Fig. 5. Male obliquebanded leafrollers captured on passive traps placed high and low in apple tree canopies in 4.1-ha pheromone-disrupted and insecticide-treated blocks during the 1st summer flight period, 1994. 


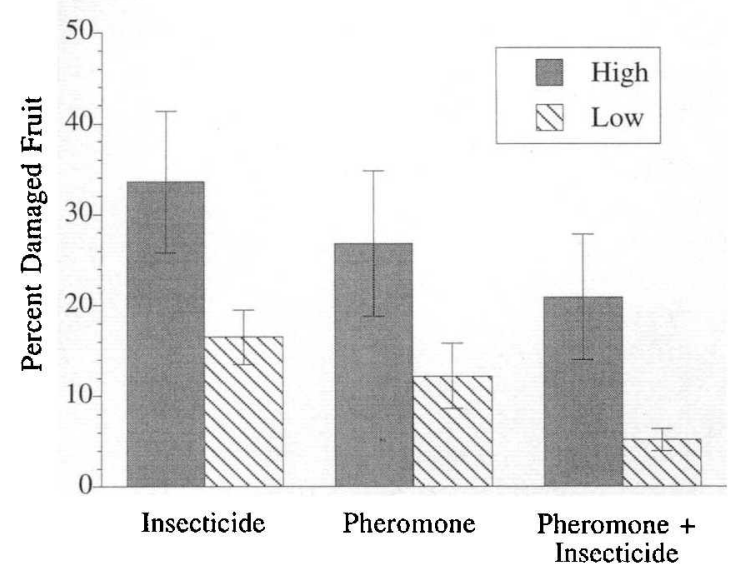

Fig. 6. Obliquebanded leafroller damaged fruit harvested from high and low positions in apple tree canopies from 3 treatments, 1994.

cantly different among the pheromone, insecticide, or pheromone plus insecticide treatments. However, in all treatments more infested terminals were located high in the tree canopy compared with low with 30.0 and $20.0 \%$ infestations occurring high and low respectively $(F=9.20$; $\mathrm{df}=1$, $12 ; P \leq 0.01)$. Fruit damage was similar among all treatments, but was significantly greater high in the tree canopy compared with low $(F=11.37$; $\mathrm{df}=$ 1,$12 ; P \leq 0.01$ ) (Fig. 6). No differences were observed among the treatments for fruit damage in any of the 4 damage rating categories (data not shown).

Edge Effect, 1994. More males were captured in pheromone-baited traps placed along the edges of the pheromone-permeated plots compared with the interior $(F=23.33$; $\mathrm{df}=1,6 ; P \leq 0.01)$ and greater trap catches were recorded from traps located high compared to low in the tree canopy ( $F$ $=35.21$; $\mathrm{df}=1,6 ; P \leq 0.01$ ) (Fig. 7). The interaction between treatment and trap location was marginally significant and appears to occur because of the increased difference between trap catches high and low in the tree canopy in the interior compared with the edge of the pheromone-permeated blocks ( $F=4.62 ; \mathrm{df}=1,6 ; P=0.07$ ) (Fig. 7). Passive trap data for male, female, and mated female moths indicate no differences between the edge and interior of pheromone disrupted blocks. of the 94 moths tethered along the edges and interior of the pheromone disrupted blocks, $55.3 \%$ were recovered alive. No mating was observed in the lower portions of the tree canopy in either the edge or interior. However, 10.0 and $13.3 \%$ of the moths located high in the canopy mated in the edge and interior respectively, but this difference was not significant.

Percentage of terminal branches infested with obliquebanded leafroller larvae was not different between the interior and edge of the pheromone plus insecticide plots. However, the upper portions

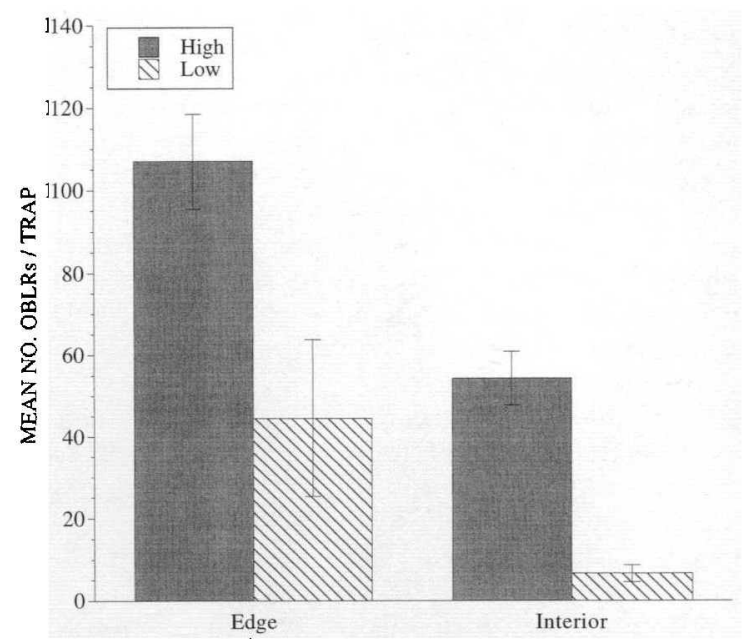

Fig. 7. Male obliquebanded leafrollers captured in pheromone-baited traps placed high and low in apple tree canopies along the edge and interior of 4.1-ha pheromone-disrupted blocks during the 1st summer flight period, 1994.

of the canopy always had greater infestation levels than did the lower canopy ( $F=8.99 ; \mathrm{df}=1,8 ; P$ $=0.02)$. No differences in fruit damage were observed between the interior and edge of the pheromone plus insecticide treatment. However, a marginal canopy position effect was observed with more damaged fruit located high compared to low in the canopy $(F=4.36$; $\mathrm{df}=1,8 ; P=0.07)$.

\section{Discussion}

Pheromone Concentration. The ability of the male obliquebanded leafroller to locate pheromone-baited traps was decreased in pheromone permeated plots. Increasing atmospheric pheromone concentration above the lowest level tested did not increase the level of disruption (Fig. 1). This indicates a threshold level above which little increase in disruption was gained. Even with the high pheromone concentrations used in our study, we were unable to completely prevent male obliquebanded leafrollers from locating pheromonebaited traps. A pheromone concentration of only 5 $\mathrm{mg} / \mathrm{h} / \mathrm{ha}$ has been reported to provide high levels of obliquebanded leafroller and redbanded leafroller disruption (Roelofs 1976, Reissig et al. 1978). Studies conducted with oriental fruit moths also indicate that no increase in disruption is gained beyond a critical atmospheric concentration (Rothschild 1975, Charlton and Cardé 1981).

Our inability to prevent males from locating pheromone-baited traps or tethered females may result from an inadequate pheromone concentration in the atmosphere at night when these animals mate. Dispenser release rates provided by the manufacture were recorded from dispensers that had been in the field during the day and night. 
Perhaps the actual nighttime release rate, when temperatures are cooler and wind speeds have decreased, are lower than what we reported. A release rate study conducted in growth chambers with the same Consep dispensers used in our 1994 study demonstrated that cool temperatures, similar to nighttime conditions, decrease the amount of pheromone released compared with release rates estimated from field collected dispensers (J.-P. Deland, Laval University, Quebec, Canada, personal observation).

Trap placement greatly influenced trap catch, with catches being greater in the top of the tree canopy (Figs. 1, 2, and 4). This suggests that mating disruption may be less effective in the upper canopy. Most studies place traps only at chest height within the tree canopy, so levels of mating disruption may be much lower in the upper portion of the tree canopy than reported.

Greater male catch in pheromone-baited traps located high in a tree canopy has been reported for other moths (Rothschild 1975, Sower and Daterman 1977, Suckling and Shaw 1992). In large pheromone-permeated blocks, there was a disproportional increase in the number of male obliquebanded leafrollers captured in pheromone traps placed high in the tree canopy compared with similarly placed traps in the comparison plots (Figs. 2 and 4). This indicates that the presence of pheromone-permeated air caused either an increase in male responsiveness to traps located high in the tree or greater inhibition of males responding to traps placed low in the canopy. This phenomenon was not observed in the pheromone rate study and may be the result of constant exposure to sex pheromone in the larger permeated plots.

Pheromone-permeated air also caused a trend of greater passive trap catch of males high in the tree canopy compared with insecticide-treated plots in 1993 (Fig. 3). However, this pattern was not significant in 1994, but significantly fewer males were captured on passive traps in the pheromone compared to the insecticide treatment, possibly indicating decreased searching movement by males in pheromone permeated plots (Fig. 5). Therefore, the presence of atmospheric sex pheromone was altering the behavior of male obliquebanded leafrollers, causing them to move toward the top of the tree canopy. Passive trap data, larval counts and fruit ratings also indicate greater obliquebanded leafroller activity in the upper tree canopy. We hypothesize that male obliquebanded leafrollers fly throughout the tree canopy in search of females. In the presence of pheromone, from a calling female, pheromone-baited trap, or pheromone dispenser, they move toward the top of the tree to locate females that are more prevalent in this position.

Codling moths have been observed to be most active in or near the top of the tree canopy (Woodside 1944). Codling moths have been observed entering apple trees and flying throughout the can- opy for 30-40 min with only brief rests (Howell et al. 1990). However, in the presence of a pheromone source suspended above the tree top, males accumulated at the top of the tree canopy. Sower and Daterman (1977) reported that the Douglasfir tussock moth, Orgyia pseudotsugata (McDunnough), respond in greater numbers to pheromone traps placed high in the canopy in the presence of pheromone-permeated air. However, in untreated plots, equal numbers of males were captured in traps placed in the high and low canopy positions. This may be a similar phenomenon to that found in our study.

Pheromone Dispenser Position. Location of pheromone dispensers within the plant canopy has been little studied, but it has been suggested as a potentially important aspect of mating disruption (Sower and Daterman 1977). Mast studies examining the effects of mating disruption deploy pheromone dispensers into 1 strata of the plant canopy without regard for where mating occurs. The ability of male obliquebanded leafrollers to locate pheromone-baited traps and tethered females in our study was not affected by pheromone dispenser placement (Fig. 2). Similar results have been obtained with male codling moths that were captured in pheromone-baited traps in similar numbers regardless of pheromone dispenser placement (Rothschild 1975).

Edge Effect. Many researchers have attributed the failure of pheromone disruption to the presence of immigrating animals and a resultant increase in fruit damage, particularly along edges of disrupted areas (Rothschild 1981, Barnes et al. 1992, Pfeiffer 1993b). The deciduous woodlands and commercial apple orchards that surrounded the orchards used in this study harbor alternative hosts for obliquebanded leafrollers (Chapman and Lienk 1971). Therefore, the potential existed for obliquebanded leafrollers to immigrate into the pheromone-disrupted blocks. It was our aim to determine if an edge effect existed around these blocks by comparing data gathered along the edges of disrupted blocks to data collected from the interior of the same blocks. Although the presence or absence of an edge effect does not directly measure immigration, if present, it would provide circumstantial evidence of increased activity along the edges of pheromone disrupted blocks.

No significant increase in the number of males, females, or gravid females captured on passive traps positioned along the edges compared with the interior of pheromone disrupted blocks was found. If obliquebanded leafrollers were moving into the disrupted blocks from adjacent habitat, an increase in the number of animals captured on passive traps positioned along the edges would be expected. The number of mated tethered females placed along the edge and interior of the pheromone treated blocks were the same. Additional information about obliquebanded leafroller movement can be gleaned from larval infestation and fruit damage, both of 
which were similar on the edge and interior of the blocks. Although the number of male moths captured in pheromone-baited traps was elevated along the edges compared with the interior of disrupted block, this probably resulted from uneven pheromone coverage along the edges, which has been reported in other pheromone disruption studies (Ogawa 1990). All of these data indicate that for obliquebanded leafrollers, no edge effect exists around pheromone disrupted orchards. It appears that most obliquebanded leafrollers originate from within commercial apple orchards or if immigration is occurring, it is accomplished by movement over the top of the edge row of trees or at levels too low for our methods to detect. The level of immigration required to increase fruit damage is unknown. However, 1 female obliquebanded leafroller is capable of producing 900 eggs (Chapman and Lienk 1971). Therefore, it may only require a few moths immigrating into a disrupted orchard to cause control failures.

Treatment Effects. The most stringent test of pheromone disruption as a pest control tactic is the effect it has on reducing crop damage (Rothschild 1981, Dennehy et al. 1991). The New York State Integrated Pest Management Program has established a treatment threshold of 3 and $10 \%$ larvalinfested terminal branches for fresh or processing fruit, respectively (Agnello et al. 1991, 1993). All treatments were well above this threshold and no different from those recorded in the pheromone treatment. This control failure was likely caused by the development of insecticide resistance that has been proliferating since the mid-1980s (Reissig et al. 1986; D.S.L., unpublished data). High larval densities caused extremely high fruit damage (Fig. 6). However, even in the presence of extremely high obliquebanded leafroller pressure, pheromone disruption performed as well as conventional insecticides. The combination of pheromone and insecticides had an additive effect on fruit damage.

Other large-plot pheromone disruption trials conducted for the control of tree fruit pests have revealed results similar to ours. The control of codling moth in apple and pear orchards using pheromone disruption has been shown to reduce fruit damage compared with untreated plots, but fruit damage in disrupted plots was often greater than that obtained from a standard insecticide program (Moffitt and Westigard 1984, Pfeiffer et al. 1993b). Oriental fruit moth damage to peaches was reduced with the use of pheromone disruption compared with that obtained with insecticide treatments (Weakley et al. 1987). Mating disruption of a leafroller complex in apples resulted in better control than in untreated control plots, but results were variable when compared with that obtained using conventional insecticides (Pfeiffer et al. 1993a). Results of pheromone disruption studies indicate the ability of pheromones to provide control of fruit damaging pests, but also reveal the variable level of control obtained with this technique.

There may be several reasons for the variable level of control obtained in our studies. We selected orchards with extremely high obliquebanded leafroller densities. High pest densities should be more difficult to disrupt than low densities because the frequency of chance encounter increases with population density. Increased levels of damage have been reported from mating disruption studies conducted in high pest-density settings (Moffitt and Westigard 1984, Dennehy et al. 1991, Pfeiffer et al. 1993a). We were unable to prevent male obliquebanded leafrollers from locating pheromone-baited traps or tethered females, even at the highest pheromone concentrations. With this ability by males to locate and mate with females during the entire season, fruit damage was inevitable. Although immigration of obliquebanded leafrollers appeared not to occur by movement through the tree canopy and into the disrupted plots, it is possible that they moved into the disrupted plots by dispersing above the apple orchard and settling uniformly into the apple trees, or immigration is limited to a few animals. If either occurred, our sampling methods would not have detected it.

Mating of tethered females in control plots during both years of this study was relatively low. Mating of tethered obliquebanded leafroller females in a mixed forest in Canada has been reported to occur in $\approx 70 \%$ of the females tested (Delisle 1995). The differences in mating frequency between this report and that obtained in our study may be the result of many factors, including tree structure and composition and leafroller densities. Forested habitats contain substantially fewer obliquebanded leafrollers than do the apple orchards used in our study because of high mortality due to natural enemies in unsprayed forested habitats (D.S.L., unpublished data). Therefore, competition for females may be greater in the smaller obliquebanded leafroller populations in forested habitats, resulting in higher mating levels. In the apple orchards used in our study, males may be more selective in choosing mates because their is less competition because of the large numbers of available females. Therefore, any abnormality in a female caused by tethering or location of the female within the tree canopy, may cause decreased levels of mating. However, the level of mating among the different treatments in our study are relative and indicate the effect of the treatments on mating of the tethered females under the conditions present during our study.

Results presented in this artical indicate that atmospheric permeation with obliquebanded leafroller synthetic sex pheromone significantly reduced male moths ability to locate pheromonebaited traps and tethered females. Inhibition of male moths to locate pheromone-baited traps was not different among 3 atmospheric pheromone 
rates, indicating a threshold level of pheromone above which no increase in mating disruption was gained. Different spatial deployment patterns of pheromone dispensers into the tree canopy did not effect mating levels. Mating of tethered females, presence of gravid feral females, high larval densities, and fruit damage within large disrupted blocks indicate the ability of obliqubanded leafrollers to continue mating in disrupted orchards. However, even under extremely high obliquebanded leafroller populations, fruit damage was similar in pheromone, pheromone plus insecticide, and insecticide treatments.

Obliquebanded leafroller activity was greatest in the upper portions of the tree canopy. Therefore, we are recommending that pheromone dispensers be placed into the upper canopy position. No edge effect was observed around the perimeter of disrupted blocks, however, an increased ability of males to locate pheromone-baited traps in this area was recorded. Therefore, we speculate that obliquebanded leafrollers are not immigrating into orchards in a manner or rate detectable in our study.

To further our understanding of pheromone disruption and to increase its efficacy, we need to conduct more detailed pheromone disruption studies. We intend to continue our studies by using largeplot (25-ha) disruption trials. This will allow better mixing and coverage of the pheromone and reduce any possible effect of immigrating moths. Small, uniform-sized trees will be used to increase uniformity of pheromone coverage by reducing pockets of unpermeated air. Orchards with a history of low to moderate obliquebanded leafroller densities will be used to decrease the frequencies of random encounters of male and female moths. Further studies are necessary to examine the feasibility of combining biorational insecticides and pheromones. With these modifications we are hopeful that synthetic sex pheromones can be used to interfere with the mate-finding communication system of the obliquebanded leafroller and provide economic control.

\section{Acknowledgments}

We acknowledge the efforts of Cindy Smith, Erin Hamill, Anna Forsline, Krista Hannacker, Mike Dunham, Joseph Kovach (Comell University), and Debbie Breth (Comell Cooperative Extension) during this study and Jim Misiti for collecting field records. We also thank Kinya Ogawa (Shin-Etsu Chemical), Don Thomson (Pacific Biocontrol) and Janice Gillespie (Consep) for providing pheromone dispensers and technical expertise. These studies could not have been conducted without the commitment of Daryl Oakes, Randy Paddock, and George Lamont who provided us the use of their orchards. Partial funding of this research was obtained from the New York State Apple Research and Development Board.

\section{References Cited}

Abacus Concepts. 1989. SuperANOVA. Abacus Concepts, Berkeley, CA.
Agnello, A. M., J. Kovach, J. P. Nyrop, W. H. Reissig, and W. Wilcox. 1991. Simplified integrated management program: guide for apple sampling procedures in New York. Comell Integrated Pest Management Bulletin 201C, Geneva, NY.

Agnello, A. M., W. H. Reissig, J. P. Nyrop, J. Kovach, and R. A. Morse. 1993. Biology and management of apple arthropods. Cornell Cooperative Extension Information Bulletin 231, Ithaca, NY.

Barnes, M. M., J. G. Millar, P. A. Kirseh, and D. C. Hawks. 1992. Codling moth (Lepidoptera: Tortricidae) control by dissemination of synthetic female sex pheromone. J. Econ. Entomol. 85: 1274-1277.

Cardé R. T., K. Trammel, and W. L. Roelofs. 1975. Disruption of sex attraction of the redbanded leafroller (Argyrotaenia velutinana) with microencapsulated pheromone components. Environ. Entomol. 4: $448-450$.

Chapman, P. J., and S. E. Lienk. 1971. Tortricid fa11na of apple in New York. N.Y. State Agricultural Experiment Station. Geneva Spec. PLbl. 87-90.

Chapman, P. J., S. E. Lienk, and R. W. Dean. 1968. Bionomics of Choristoneura rosaceana. Ann. Entomol. Soc. Am. 61: 285-290.

Charlton, R. E., and R. T. Cardé. 1981. Comparing the effectiveness of sexual communication disruption in the Oriental fruit noth (Grapholita molesta) using different combinations and dosages of its pheromone blend. J. Chem. Ecol. 7: 501-508.

Deland, J.-P., G. J. R. Judd, and B. D. Roitberg. 1994. Disruption of pheromone communication in three sympatric leafroller (Lepidoptera: Tortricidae) pests of apple in British Columbia. Environ. Entomol. 23: 1084-1090.

Delisle, J. 1992. Age related changes in the calling behavior and the attractiveness of obliquebanded leafroller virgin females, Choristoneura rosaceana, under different constant and fluctuating temperature conditions. Entomol. Exp. Appl. 63: 55-62.

1995. Effect of male and female age on the mating success of the obliquebanded leafroller Choristoneura rosaceana (Lepidoptera: Tortricidae) under different ecological conditions. J. Insect Behavior 8: 781-799.

Delisle, J., and L. Royer. 1994. Changes in pheromone titer of oblique-banded leafroller, Choristoneura rosaceana, virgin females as a function of time of day, age, and temperature. J. Chem. Ecology. 20: 4569.

Dennehy, T. J., L. G. Clark, and J. S. Kamas. 1991. Pheromonal control of the grape berry moth: an effective alternative to conventional insecticides. N.Y. State Agricultural Experiment Station, Geneva New York's Food and Life Science Bull. 135.

Hathaway, D. O. 1979. Codling moth: tethering females to determine mating in field populations. U.S. Dep. Agric. Sci. Educ. Admin. Adv. Agric. Technol. AAT-W-6.

Hill, A. S., and W. L. Roelofs. 1979. Sex pheromone components of the obliquebanded leafroller moth. J. Chem. Ecol. 5: 3-11.

Howell, J. F., R. S. Schmidt, D. R. Horton, S. U. K. Khattak, and L. D. White. 1990. Codling moth: male moth activity in response to pheromone lures and pheromone-baited traps at different elevations within and between trees. Environ. Entomol. 19: 573577.

Howell, J. F., A. L. Knight, T. R. Unruh, D. F. Brown, J. L. Krysan, C. R. Sell, and P. A. Kirseh. 1992. 
Control of codling moth in apple and pear with sex pheromone-mediated mating disruption. J. Econ. Entomol. 85: 918-925.

Kehat, M., S. Gothilf, E. Dunkelblum, N. Bar Shavit, and D. Cordon. 1985. Night observations on the cotton leafworm, Spodoptera littoralis: reliability of pheromone traps for population assessment and efficiency of widely separated pheromone dispensers for mating disruption. Phytoparasitica 13: 215-220.

Marks, R. J. 1976. The influence of behavior modifying chemicals on mating success of the red bollworm Diparopsis castanea Hmps. (Lepidoptera, Noctuidae) in Malawi. Bull. Entomol. Res. 66: 279-300.

Moffitt, H. R., and P. H. Westigard. 1984. Suppression of the codling moth (Lepidoptera: Tortricidae) population on pears in Southem Oregon through mating disruption with sex pheromone. J. Econ. Entomol. 77: 1513-1519.

New York State Department Agriculture and Marketing. 1973. New York State apple grades: rules and regulations. N.Y. State Dep. Agric. Mktg. Circ. 859.

Oguwa, K. 1990. Commercial development: mating disruption of tea tortrix moths, pp. 547-551. In R. L. Ridgway, R. M. Silverstein, and M. N. Inscoe [eds.], Behavior-modifying chemicals for insect management: applications of pheromones and other attractants. Marcel Dekker, New York.

Onstad, D. W., W. H. Reissig, and C. A. Shoemaker. 1985. Phenology and management of the obliquebanded leafroller (Lepidoptera: Tortricidae) in apple orchards. J. Econ. Entomol. 78: 1455-1462.

Pfeiffer, D. G., W. Kaakeh, J. C. Killian, M. W. Lachance, and P. Kirsch. 1993a. Mating disruption to control damage by leafrollers in Virginia apple orchards. Entomol. Exp. Appl. 67: 47-56.

1993b. Mating disruption for control of damage by codling moth in Virginia apple orchards. Entomol. Exp. Appl. 67: 57-64.

Reissig, W. H. 1978. Biology and control of the obliquebanded leafroller on apples. J. Econ. Entomol. 71: 804-809.

Reissig, W. H., M. Novak, and W. L. Roelofs. 1978. Orientation disruption of Argyrotaenia velutinana and Choristoneura rosaceana male mothis. Environ. Entomol. 7: 631-632.

Reissig, W. H., B. H. Stanley, and H. E. Hebding. 1986. Azinphosmethyl resistance and weight-related response of obliquebanded leafroller (Lepidoptera: Tortricidae) larvae to insecticides. J. Econ. Entomol. 79: 329-333.
Ridgway, R. L., R. M. Silverstein, and M. N. Inscoe. 1990. Behavior-modifying chemicals for insect management: application of pheromones and other attractants. Marcel Dekker, New York.

Roelofs, W. L. 1976. Communication disruption by pheromone components, pp. 123-134. In Proceedings, Insect Pheromone and Their Applications, 8-11 December 1976. National Institute of Agricultural Science, Ministry of Agriculture and Forestry, Nishigahara, Kita-ku, Tokoyo.

Roelofs, W. L., and M. A. Novak. 1981. Small-plot disorientation tests for screening potential mating disruptants, pp. 229-242. In E. R. Mitchell [ed.], Management of insect pests with semiochemicals. Plenum, New York.

Roelofs, W. L., and J. P. Tette. 1970. Sex pheromone of the oblique-banded leafroller moth. Nature (Lond.) 226: 1172 .

Rothschild, G.H.L. 1975. Control of Oriental fruit moth (Cydia molestn (Busck) (Lepidoptera, Tortricidae)) with synthetic female pheromone. Bull. Entomol. Res. 65: 473-490.

1981. Mating disruption of lepidopterous pests: current status and future prospects, pp. 207-228. In E. R. Mitchell [ed.], Management of insect pests with semiochemicals. Plenum, New York.

Shorey, H. H., and R. L. Hale. 1965. Mass rearing of the larvae of nine noctuid species on a simple artificial medium. J. Econ. Entomol. 58: 522-524.

Sower, L. L., and G. E. Daterman. 1977. Evaluation of synthetic sex pheromone as a control agent for Douglas-fir tussock moths. Environ. Entomol. 6: 889892.

Suckling, D. M., and P. W. Shaw, 1992. Conditions that favor mating disruption of Epiphyas postvittana (Lepidoptera: Tortricidae). Environ. Entomol. 21: 949-956.

Weakley, C. V., P. Kirsch, and R. E. Rice. 1987. Control of oriental fruit moth by mating disruption. Calif. Agric. May-June: 7-8.

Weissling, T. J., and A. L. Knight. 1994. Passive trap for monitoring codling moth (Lepidoptera: Tortricidae) flight activity. J. Econ. Entomol. 87: 103-107.

Woodside, A. M. 1944. Codling-moth infestation at different heights in apple trees. Virginia Agricultural Experiment Station. Bulletin 360, Blacksburg, VA.

Received for publication 21 July 1995; accepted 1 April 1996. 